\section{Asthma: Sieben Kriterien helfen auf die Spur}

Bei Patienten mit Atemwegsbeschwerden unklarer Genese lässt sich anhand einer Sieben-Punkte-Diagnostik klären, ob ein Asthma bronchiale vorliegt.

Die "Global Initiative for Asthma“ (GINA) hat letztes Jahr ein Update ihrer Leitlinie zu Management und Prävention von Asthma veröffentlicht. Um die Umsetzung im hausärztlichen Alltag zu erleichtern, haben Pneumologen um Dr. Ekkehard Beck, Rüdersdorf, die wichtigsten Schritte in einem einfachen Behandlungspfad zusammengefasst (Atemwegsund Lungenkrankheiten 2016;42:14-24).

Dr. Martin Kohlhäufl, Klinik Schillerhöhe in Stuttgart, hat ihn beim Praxis Update in München vorgestellt. Wenn ein Patient mit Atemwegsbeschwerden unklarer Genese in die Praxis kommt, empfehlen die Experten eine SiebenPunkte-Diagnostik. Verdächtig auf ein Asthma bronchiale sind:

- Trockener Husten am Tag,

- Aufwachen mit Husten in der Nacht/ am Morgen,

- Giemen beim Atmen,

- Brustenge/Atemnot,

- Verstärkung der Symptome bei Virusinfektionen, feuchtkalter Luft sowie bei körperlicher Belastung,

- Verlängertes Exspirium oder Giemen bei der Auskultation,

- Eine $\mathrm{FEV}_{1}<80 \%$ vom Soll in der Spirometrie (nur bei positivem Auskultationsbefund empfohlen).

„Wenn von den sieben genannten Punkten mindestens drei erfüllt sind, weist das in Richtung Asthma und rechtfertigt die Einleitung einer Asthmatherapie“, sagte Kohlhäufl. Dazu soll der Patient, zunächst befristet auf vier Wochen, ein kurz wirksames Betamimetikum erhalten. „Wenn der Patient das mehr als dreimal pro Woche braucht, müssen Sie direkt in die Basistherapie mit einem niedrig dosierten inhalativen Kortikosteroid (ICS) einsteigen."

\section{Bei schwerer Symptomatik zum Pneumologen überweisen}

Wenn Symptome nachts und am Morgen fehlen, rät Kohlhäufl, einen Pneumologen hinzuziehen, um andere Diagnosen abzuklären. Direkt an den Facharzt überwiesen werden sollten Patienten mit schwerer Symptomatik, einer $\mathrm{FEV}_{1}<60 \%$ vom Soll und unveränderter Symptomatik trotz einer Therapie mit inhalativem Kortikosteroid plus lang wirksamem Betamimetikum.

Als kontrolliert ist ein Asthma laut GINA anzusehen, wenn die Patienten höchstens zweimal pro Woche tagsüber Symptome haben, nachts nicht wegen ihres Asthmas aufwachen, höchstens zweimal pro Woche ein Notfallspray anwenden und in ihren Aktivitäten nicht eingeschränkt sind. Zum Erreichen der Asthmakontrolle soll die Therapie stufenweise eskaliert werden.

Dr. Beate Schumacher

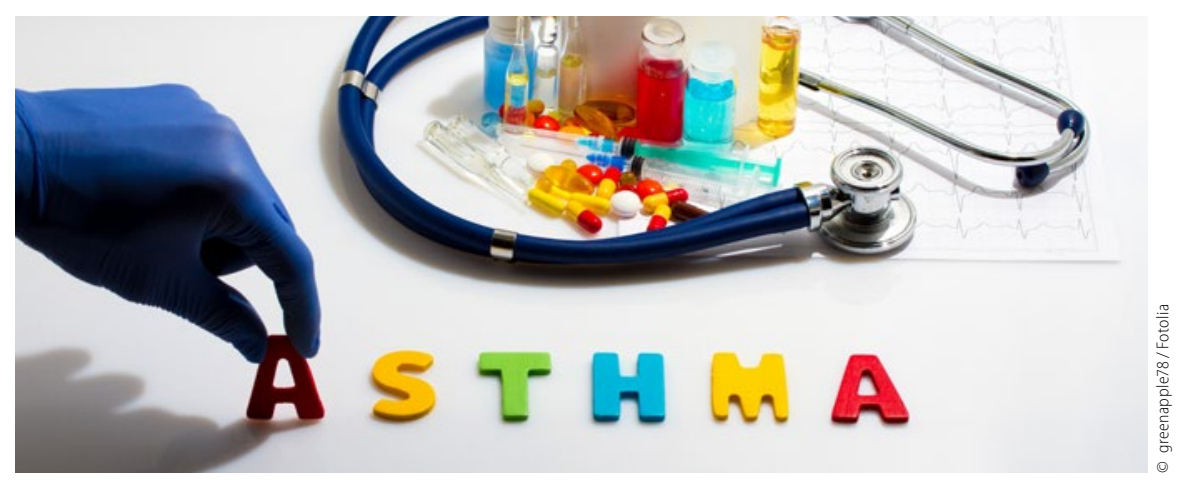

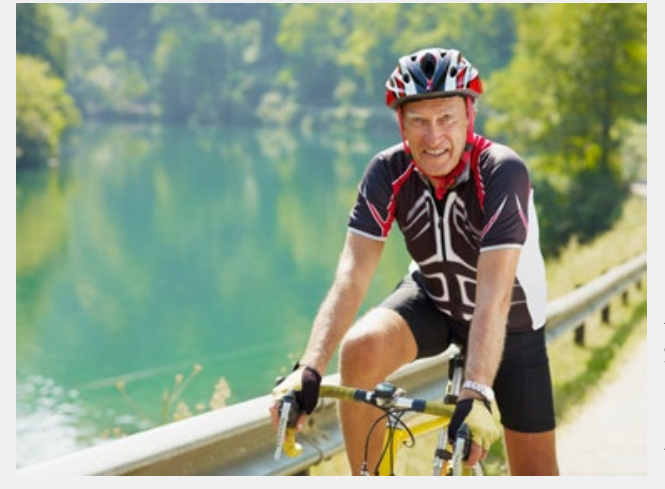

\section{Fitness schützt vor Lungenfunktionsverlust im Alter}

Wenn das kein Grund ist, den inneren Schweinhund zu überwinden: Wer körperlich fit ist und fit bleibt, hat eine deutlich geringere altersbedingte Abnahme der Lungenfunktion als körperlich weniger aktive Zeitgenossen, berichten Forscher um Dr. Lillian Benck von der Feinberg School of Medicine in Chicago. Wer körperlich hingegen stark abbaut, erleidet auch einen besonders deutlichen Abfall der Lungenfunktion im Alter.

Ein Abfall der Lungenfunktion erhöht die Morbidität und Mortalität im Alter, auch wenn der Patient keine spezifische Lungenerkrankung aufweist, betonen die Autoren.

Sie hatten im Rahmen der CARDIA-Studie circa 5.000 Gesunde im Alter von 18 bis 30 Jahre nachverfolgt und dabei regelmäßig das Fitness-Niveau sowie die Lungenfunktion ( FEV $_{1}$, FVC) ermittelt.

\section{Asthma bronchiale überdiagnostiziert}

Nach Befunden einer prospektiven Kohortenstudie aus Kanada wird Asthma bronchiale beim Allgemeinarzt überdiagnostiziert. Die Autoren um Dr. Shawn Aaron von dem Ottawa Hospital Research Institut hatten 701 Patienten mit Asthma-Diagnose durch den Hausarzt diagnostisch unter die Lupe genommen (Spirometrie, Symptomquantifizierung, bronchiale Provokationstests) und anschließend die AsthmaMedikamente ausgeschlichen.

Wer daraufhin keine Symptome, keine reversible Bronchokonstriktion und keine Hyperreagibilität zeigte, wurde als Fehldiagnose eingestuft. Dies war bei 37,5\% der Patienten der Fall. $30 \%$ benötigten auch 12 Monate später keine Asthma-Medikamente. Bei $87 \%$ dieser Patienten wurden alternative Diagnosen gestellt.

Die hausärztlichen Asthma-Diagnosen waren zu $50 \%$ ohne Spirometrie erfolgt.

de

Quelle: American Thoracic Society International Conference, San Francisco 13-18. Mai 2016 